\title{
ON THE MINIMUM NUMBER OF DISTINCT EIGENVALUES FOR A SYMMETRIC MATRIX WHOSE GRAPH IS A GIVEN TREE
}

\author{
ANTÓNIO LEAL-DuARTE AND CHARLES R. JOHNSON
}

\begin{abstract}
It is shown that for any tree $T$ the minimum number of distinct eigenvalues of an Hermitian matrix whose graph is $T$ (diagonal entries free) is at least the number of vertices in a longest path of $T$. This is another step toward the general problem of characterizing the possible multiplicities for a given graph. Related observations are made and the result facilitates a table of multiplicities for trees on fewer than 8 vertices.
\end{abstract}

Mathematics subject classification (2000): 15A18, 15A57, 05C50, 05C05, 05C12.

Key words and phrases: Graph, tree, matrices, eigenvalues.

\section{REFERENCES}

[1] R. BRualdi AND H. J. RYSER, Combinatorial Matrix Theory, University Press, New York, 1991.

[2] W. Ferguson, The Construction of Jacobi and Periodic Jacobi Matrices with Prescribed Spectra, Math. Comp. 35 (1980), 1203-1220.

[3] C. GodsIL, Algebraic Combinatorics, Chapman and Hall, New York, 1993.

[4] R. Horn And C. R. Johnson, Matrix Analysis, Cambridge University Press, 1985.

[5] C. R. Johnson AND A. LeAL-Dunte, The Maximum Multiplicity of an Eigenvalue in a Matrix Whose Graph is a Tree, Linear and Multilinear Algebra 46 (1999), 139-144.

[6] S. PARTER, On the Eigenvalues and Eigenvectors of a Class of Matrices, J. Soc. Indust. Appl. Math. 8 (1960), 376-388.

[7] G. WIENER, Spectral Multiplicity and Splitting Results for a Class of Qualitative Matrices, Linear Alg. Appl. 61 (1984), 15-29. 